Research Article

\title{
An Integrated Decoupling Device for Azimuth Control of a Balloon-Borne Gondola
}

\author{
Honghui Wang, Xiaojun Yu $\mathbb{D}^{D}$, Juan Wu, Shicheng Liang, Zeming Fan, and Zhaohui Yuan
}

School of Automation, Northwestern Polytechnical University, Xi'an 710129, Shaanxi, China

Correspondence should be addressed to Xiaojun Yu; e070035@e.ntu.edu.sg

Received 28 June 2020; Accepted 5 August 2020; Published 25 August 2020

Academic Editor: Łukasz Jankowski

Copyright (C) 2020 Honghui Wang et al. This is an open access article distributed under the Creative Commons Attribution License, which permits unrestricted use, distribution, and reproduction in any medium, provided the original work is properly cited.

\begin{abstract}
Controlling and maintaining the orientation of the balloon-borne gondola for high-altitude flight is a prerequisite for ensuring the pointing control of observation instruments. When the balloon-borne gondola is flying in the stratosphere of the atmosphere, the existing external interferences will be converted into the coupling moment to the azimuth control system. Meanwhile, those uncertain factors and the frictional nonlinearity of the control system will also cause a certain magnitude of coupling moment. The existence of such coupling moment largely impacts on the accuracy and stability of the orientation control for the angular momentum exchange devices of the balloon-borne gondola. To address such an issue, this paper proposes and implements a novel type of integrated decoupler device. With this decoupler adopted, the aziDmuth control system could sense the existence of coupling torque and azimuth fluctuations quickly and suppress the influences of external interference, uncertain factors, and system structure nonlinearity on the azimuth control effectively, thereby improving the control accuracy of the azimuth control system. Both simulations and experiments are conducted to verify the effectiveness of the proposed device. The results show that the integration of the decoupler and the controller of the azimuth control system provide the azimuth control of the balloon-borne gondola with high accuracy and stability. Such a decoupler device design has a broad potential and could not only be used for balloon-borne gondola control but also could be applied onto other control systems using angular momentum exchange devices as actuators.
\end{abstract}

\section{Introduction}

The balloon-borne gondola is a carrying platform used to carry out relevant scientific experiments for observational instruments [1]. It is typically suspended below a balloon with a sling of about $10 \sim 100 \mathrm{~m}$ and is launched into the stratosphere together with the balloon at a flying height of about $30 \mathrm{~km}$ to $40 \mathrm{~km}$. When the balloon-borne gondola platform is flying at the high altitude, it has to maintain a relatively stable orientation to meet the desired pointing from the observational instrument to the target $[2,3]$. Therefore, in order to achieve the desired pointing control of the observational instrument, it is necessary to realize stable orientation control for the balloon-borne gondola platform $[2,4]$.
The conventional balloon-borne gondola azimuth control system usually adopts a reaction flywheel as an angular momentum exchange device, which controls the angular acceleration changes to generate a control torque to drive the momentum moment of the gondola and helps realize the rotation control of the ball-borne gondola along the vertical direction. For example, Huguenin et al. presented the first stratospheric balloon-borne platforms for infrared astrophysical observations [5]; Villela et al. described a system design for the attitude control of a balloon-borne gamma ray telescope based on the reaction wheel and tested its performances [6], while Ye et al. reported the attitude control and high-precision pointing control system for a large balloon-borne solar telescope [7]. In recent years, there have also been some studies on either the control system design 
$[2,8]$ or the overall platform implementations $[9,10]$ for balloon-borne experiments and observations.

Although those reported systems achieved satisfactory control precision, there still exist a number of problems after integrating the azimuth control system onto the ball-carrying gondola platform, however, due to the various external factors and the inherent system nonlinearities [11, 12]. First, the external disturbances, such as the unequal wind pressure, could drive the balloon to rotate along the plumb line and generate coupling torque between the hanging rope and the gondola [13]. Such coupling torque would saturate the flywheel's velocity, driving the azimuth control system to be uncontrollable [14]. This is also one of the inherent characteristics of the angular momentum exchange devices. Second, the disordered weak airflow and the other uncertain factors can also introduce coupling torques between the hanging rope and the gondola. Meanwhile, since the azimuth control system is a underdamped system, the fluctuation of the coupling torque would cause the orientation of the balloon-borne gondola to vary easily, which thus impacts on the system control accuracy and stability $[13,15]$. Last but not least, during the transient state when the gondola orientation is modified at its transient state, the gondola azimuthal control may fail due to the frictional nonlinearity of the system structure, and such a state may last for a while until the influences of the frictional torque are eliminated with the increased control torque $[16,17]$. Such an issue caused by the transient state would largely reduce the system control accuracy and agility [18].

In order to solve the above problems and reduce the burden of the azimuth controller, it is necessary to design appropriate decouplers that enable the azimuth controller to quickly perceive and eliminate the coupling moment caused by uncertain factors first and then isolate the influences of the coupling moment on the azimuthal control system [19]. Meanwhile, the decoupler is desired to compensate the coupling torque caused by the frictional nonlinearity of the control system structure such that the available control torque of the azimuth control system can be extracted more timely $[20,21]$. In this way, the influence of coupling moment on angular momentum exchange system could be effectively suppressed to maintain the best operating point for the system $[22,23]$.

In this paper, we analyze the formation and characteristics of the coupling torque, as well as its influences to the azimuth control of the balloon-borne gondola first and then propose an integrated decoupling device for azimuth control. Both simulations and experiments are finally conducted to verify the effectiveness of the proposed decoupling device. The results show that an integration of the decoupler and the azimuth control system could help realize highly stable and accurate azimuth control for the balloon-borne gondola. The main contributions of our study could be summarized into two aspects: first, both the formation and the influences of the coupling torque introduced by the nonlinearities and uncertain disturbances are theoretically analyzed when the balloon-borne gondola is working in the air, and secondly, an integrated decoupler that could help avoid velocity saturation of the flywheel motor is proposed and experimentally verified, which thus lays a good foundation for the azimuth control of the balloon-borne gondola.

\section{Coupling Torque Analysis}

2.1. Formation and Characteristics of Coupling Torque. In the balloon-borne gondola system, the gondola is suspended below the balloon using four tenacious nylon ropes. During its ascension and floating process, the balloon may rotate under the influences of the wind pressure. Specifically, such a rotational motion typically occurs during the rising process owing to the irregular balloon shape, while the rotation direction rarely changes. Once the balloon rises up to the stratosphere and enters a stable horizontal flight state, the wind pressure around the balloon may become unequal due to the slight air turbulence [24], which thus generates a random force around the balloon, causing it to rotate slowly and periodically with a variable velocity. Due to the inertia of the balloon and the balloon-borne gondola below the ropes, however, such two kinds of balloon rotations will cause the rope to twist gradually. Figure 1 shows the sling rope distortion and forces experienced by the balloon. As can be seen, when the four suspending ropes are in a twisted state, they could act as a torsion spring and generate torque, which could be calculated as follows [9]:

$$
T_{s}=k_{s}(r) \cdot \theta \text {, }
$$

where $k_{s}(r)$ is the equivalent torsional coefficient of the suspending rope, $r$ is the number of rounds that the four ropes have turned, and $\theta$ is the tiny angle of the relative motion between the balloon and the gondola in any given state. The transient value of $k_{s}(r)$ is proportional to $r$, yet the longer the slings are, the smaller the torsion coefficients are.

In practice, when the torque is large enough, it would drive the balloon-borne gondola to rotate and enter a new equilibrium state $[14,25]$. Under the influences of the balancing torsional moment, the rotation period of the suspending gondola is almost the same as that of the balloon. Furthermore, a small amount of airflow disorder and the other factors could also impact on the gondola and, thus, generate an indeterminate torsional moment $T_{a}$ around the plumb line. In such a case, $T_{a}$ and $T_{s}$ together would impact on the gondola in the form of a disturbance torque $T_{d}$, which is in the vector form satisfying a relationship as follows:

$$
\vec{T}_{a}+\vec{T}_{s}=\vec{T}_{d}
$$

In equation (2), the size of $T_{s}$ changes periodically with its frequency determined by the speed at which the balloon drives the gondola to rotate. Such a torsional moment always exists during the flight of the gondola if no actions are taken to eliminate or reduce it. In practice, since the direction of $T_{a}$ is not fixed, it may either counteract the effect of $T_{s}$ or increase $T_{s}$. However, since $T_{a}$ appears randomly and it is also much smaller as compared with $T_{s}$, the disturbance moment $T_{d}$ is mainly determined by $T_{s}$.

The coupling torque caused by the frictional nonlinearity of the system structure impacts on the gondola in the form of internal interference [26]. According to the Dahl friction 


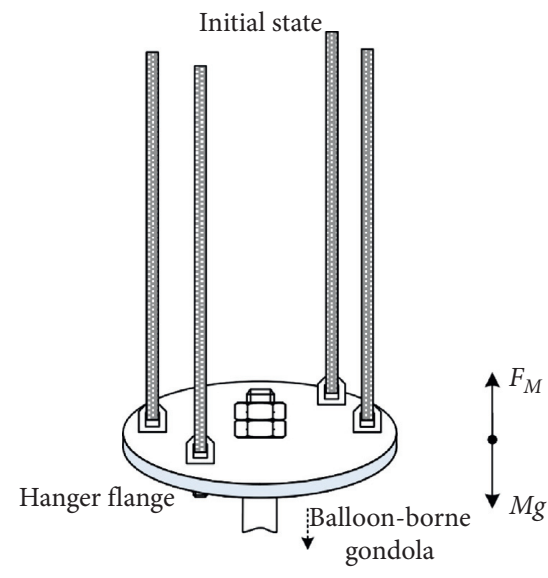

(a)

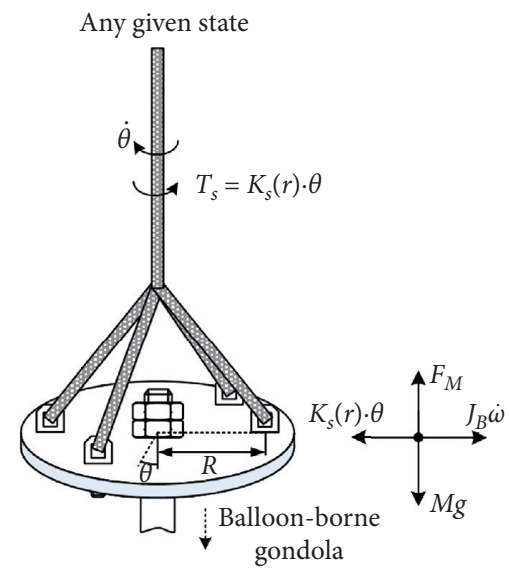

(b)

FIGURE 1: Sling rope distortion and force diagram for a balloon-borne gondola at its initial state (a) and any state driven by a torque (b).

model, the friction model could be expressed as follows $[27,28]$ :

$$
\left\{\begin{array}{l}
\dot{T}_{f}=\beta \dot{\theta}\left[T_{f} \operatorname{sgn}(\dot{\theta})-T_{c}\right]^{2}, \\
T_{b}=\ddot{\theta},
\end{array}\right.
$$

where $\dot{\theta}$ is the relative angular velocity between the balloon and the gondola, $T_{f}$ is the dynamic friction torque, while $T_{b}$ and $T_{c}$ are the viscous friction torque and coulomb friction torque, respectively.

Once there is a compensation torque $T_{m}$, they should satisfy the following relationship:

$$
\dot{\theta}=-\frac{1}{J}\left(T_{m}-T_{b}-T_{f}\right)
$$

where $J$ is the equivalent inertia of the gondola.

2.2. Influence of Coupling Torque. Assume that the balloonborne gondola azimuth control system adopts a reaction flywheel as an angular momentum exchange device. Figure 2 shows a schematic diagram for the working principle of the reaction flywheel.

According to the momentum moment conservation law, the derivative of the sum of momentum vector of the ballborne gondola on its rotation axis equals to the sum of the external moment vectors acting on the balloon-borne gondola. Assuming the initial angular velocity of the flywheel is $\omega_{0}$, one can have

$$
\frac{\mathrm{d}}{\mathrm{d} t}\left(L_{w}+L_{b}\right)=T_{d}
$$

While the momentum moments of the flywheel and the gondola (excluding the flywheel) are $L_{w}=J_{w}(\omega+\dot{\psi})$ and $L_{b}=\left(J_{g}-J_{w}\right) \dot{\psi}$, respectively, then the following equation holds:

$$
J_{w}\left(\omega-\omega_{0}\right)+J_{g} \dot{\psi}=\int_{0}^{t} T_{d} \mathrm{~d} t,
$$

where $J_{w}$ is the equivalent inertia of the flywheel, $J_{g}$ is the total equivalent inertia of the gondola and the flywheel, $\psi$ is the deviation angle between the gondola's azimuth and the expected azimuth, and $\omega$ and $\omega_{0}$ are the angular velocity and initial angular velocity of the flywheel, respectively.

In order to stabilize the orientation of the gondola, that is, the angle does not change with $\dot{\psi}=0$, it is necessary to eliminate the influence of the external interference torque $T_{d}$, and therefore, the flywheel speed should change according to the following equation:

$$
\omega=\omega_{0}+\frac{1}{J_{w}} \int_{0}^{t} T_{d} \mathrm{~d} t .
$$

The above equation indicates that when the flywheel with angular inertia $J_{w}$ accelerates (or decelerates) from $\omega_{0}$ to $\omega$ in a unit time, it could generate a reaction moment $T_{w}=-T_{d}$ to counteract with the disturbing moment $T_{d}$. During this period of time, although the balloon-borne gondola is affected by the disturbing moment, it does not rotate, while the magnitude of the azimuth deviation angle does not change either, and therefore, the orientation of the gondola is in a stable state. However, since the angle of azimuth deviation caused by the interference torque $T_{d}$ is not reduced or eliminated, the flywheel still has to accelerate further to provide the desired control torque to maintain the gondola rotate in the opposite direction until the angle of azimuth deviation is reduced to zero, i.e., $\psi=0$. Such is the purpose of azimuth control.

Equation (7) also indicates that if the disturbance torque $T_{d}$ could not be eliminated, the flywheel will maintain a certain angular acceleration until its velocity saturates, while the orientation of the gondola will be out-of-control. When the interference torque $T_{d}$ is zero, the flywheel's optimal working point could be set to the state of zero rotation rate, i.e., $\omega_{0}=0$. When the flywheel starts to rotate from the state 


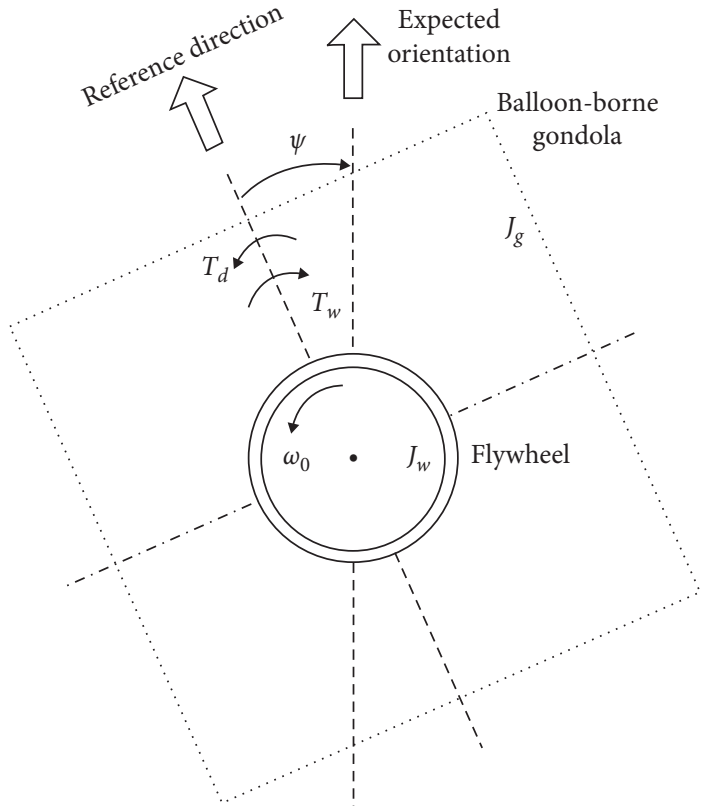

FIGURE 2: Schematic diagram illustrating the working principle of reaction flywheel.

of zero rotation speed at a certain acceleration, the available control torque can be generated to directly drive the gondola to rotate.

In addition, in equation (2), there also exists an uncertain term in the external interference torque $T_{d}$ with a small magnitude, and it may cause fluctuations in the flywheel velocity. Since the azimuth control system is underdamped, such fluctuations can easily drive the control process to oscillate, which thus increases the azimuth deviation. Due to the friction moment in the control system, when the orientation of the ball-borne gondola needs to be corrected, there would be a transient out-of-control state at the time when there is a relative movement between the gondola and the balloon. Only after the control torque generated by the flywheel offsets the friction torque, the control system could then generate a control torque $T_{g}$, which could be used to adjust the orientation of the gondola. It is worth noting that the residual friction torque $T_{f}$ may also cause the flywheel speed to become saturated. Figure 3 shows the simplified schematic diagram for the working principle of the reaction flywheel azimuth control.

2.3. Integrated Decoupling Design. As shown in Figure 3, one could find that the main objective of the decoupler design is to eliminate the influence of the coupling torque and then convert all the control torques $T_{w}$ output by the flywheel into control torques $T_{g}$ used for controlling the rotation of the gondola directly [29]. In such a way, a higher azimuth control accuracy can be obtained by appropriate parameter setting and/or correction for the azimuth controller.

The decoupler design consists of the following three steps. Firstly, design a decoupling mechanism to reduce the torsional interference torque of the suspending rope to a certain magnitude [29] and then adopt the decoupling mechanism-based torque generator to generate the desired torque to compensate or offset the coupling torque caused by external interference [30]. Since the weak moment caused by uncertain factors is not easy to be detected by hardware, the final step is to devise a state observer to identify the existence of the coupling moment. Meanwhile, the devised observer can also compensate the friction moment to make the extraction of the available control moment timely.

2.4. Decoupling Mechanism Design. Figure 4 shows the hardware structure schematic diagram of the proposed decoupler for the balloon-borne gondola. As seen, it is composed of a bearing, a torque converter, a torque motor, and some other main components.

In such a devise, the sling flange plate and the gondola could rotate relatively due to the existence of the bearing under the sling flange plate, and therefore, when the balloon rotates, the sling rope will not be twisted too tightly, while the magnitude of $T_{s}$ could be greatly reduced. Meantime, as the residual $T_{s}$ value can be measured by the torsional converter, it could be used as feedback to obtain the calculation errors. Such errors could be further amplified by an amplifier to drive the torque motor, which finally formulates a force servo loop. The torque motor outputs a compensating torque to offset $T_{s}$, and thus, it eliminates the influences of $T_{s}$.

Theoretically, as long as the torque reference value of the force servo closed loop is set to be zero, the decoupler could eliminate the influence of external disturbance torque with the generated additional torque, and thus, the flywheel operating point is set to the zero velocity state [31]. In practice, however, it is very difficult to totally eliminate the torque on the torsional converter, and therefore, the speed of the flywheel could also tend to saturate as long as there is still a residual coupling torque on the torsional converter. In addition, although it is difficult to detect the weak torque caused by the uncertain factors and the friction torque of mechanical structure through the torsional converter, these existing torques would introduce changes in the flywheel rotation speed, which thus could affect both the accuracy and agility of azimuth control.

2.5. Integrated Decoupler. To address the abovementioned problems, a decoupler scheme, as shown in Figure 5, is proposed in this paper.

In Figure 5, it is assumed that the flywheel stops rotating with the output control torque is set to be zero. The actual influence factors on the orientation of the gondola are the sum of the adjustment torques $T_{s}$ and $T_{a}$ generated by the superposition of the balloon angle, decoupling angle, and gondola angle on the hanging rope. The external interference input could cause azimuth deviation of the gondola, and such deviation signal could be processed by the azimuth controller and recognized by the state observer. This deviation signal is input into the decoupler as a reference torque. By utilizing a force servo closed-loop system, the decoupler could adjust the torque $T_{s}$ of the suspending rope quickly. The adjusted torque could act on the gondola and drive the 


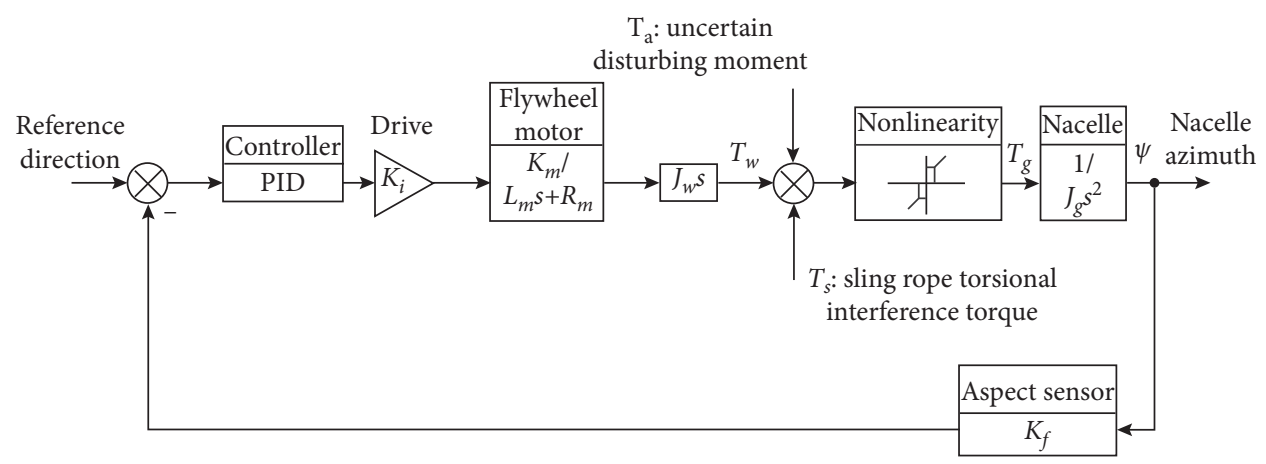

FIgURE 3: The simplified schematic diagram illustrating the working principle of the reaction flywheel azimuth control.

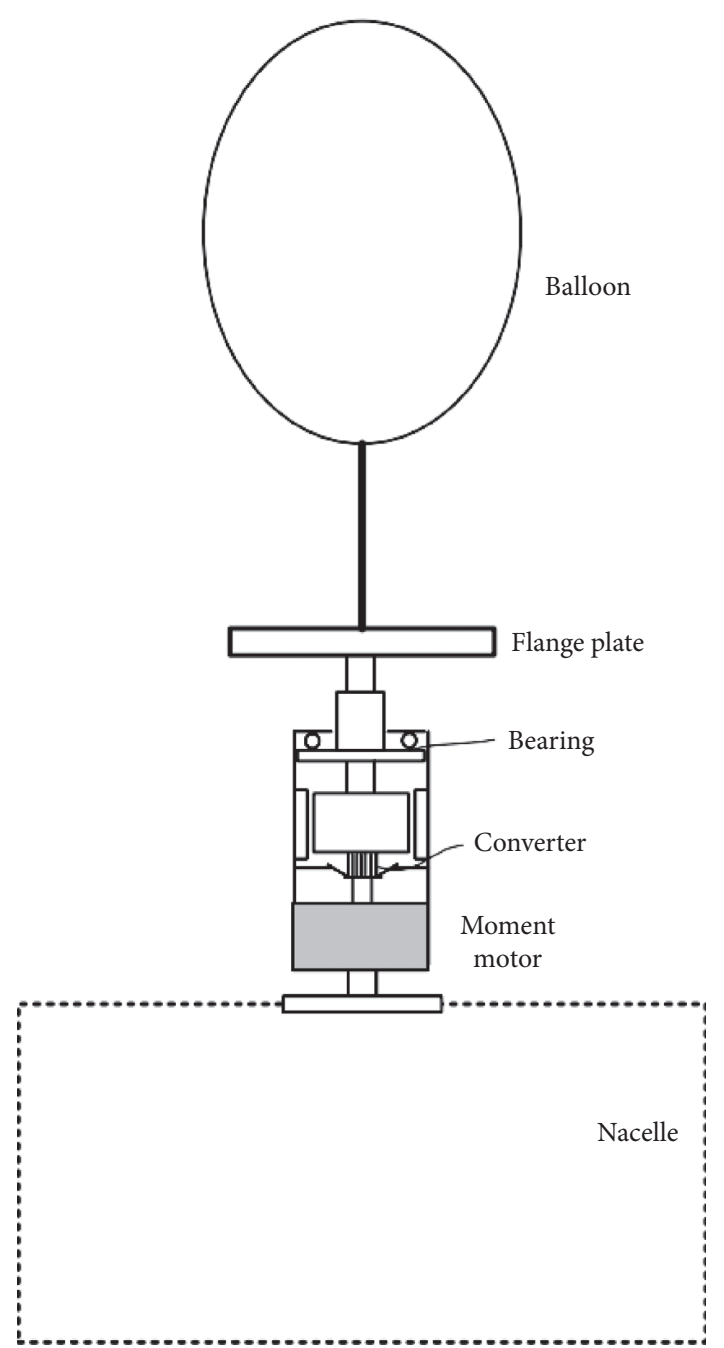

FIgURE 4: The simplified schematic diagram of hardware system for the proposed decoupler.

gondola to rotate until the azimuth deviation is corrected near to zero. The decoupler will stop torque adjustment once the input reference torque approaches zero.

By utilizing this adjustment process, the decoupler transforms the problem of detecting the weak moment and nonlinear compensation to the problem of observing and controlling the azimuth deviation of the gondola, which thus simplifies the complexity of the problem. In addition, by monitoring the flywheel velocity, the decoupler could also adjust the reference torque appropriately to accelerate the whole adjustment process.

According to the above analysis, the decoupler itself could achieve azimuth control via torque adjustment, and therefore, the flywheel does not need to rotate to generate adjusting torque, and its working speed can be stabilized near zero. Since the inertia of the gondola is large, the azimuth can only be adjusted slowly. The agility of the control system can be improved by adjusting the torque applied on the gondola shaft.

Since the power of the torque motor of the decoupler is quite small, it is difficult for the decoupler generated torque to drive the gondola to rotate. Therefore, in engineering practice, the decoupler torque motor and the flywheel run synchronously. When the azimuth deviation is large, the flywheel has a large acceleration, while the decoupler can output a large compensation torque. Once the azimuth deviation decreases, the acceleration of the flywheel and the output torque of the decoupler will converge.

\section{Simulations and Experiments}

3.1. Simulations. According to the existing test data, due to the presence of the decoupler bearing, the existing test data show that the disturbing torque that acts on the gondola and caused by the twisted hanging rope is about $2 \mathrm{Nm}$, and the friction torque of the bearing is about $0.6 \sim 0.9 \mathrm{Nm}$. Simulations are conducted to verify the effectiveness of the proposed integrated decoupler device on a personal workstation with AMD Ryzen $526003.40 \mathrm{GHz}$ processor, 64 bit Windows OS, and $16 \mathrm{~GB}$ RAM using Matlab/Simulink R2018a. The parameters utilized for simulations are shown as follows.

Table 1 presents the numerical values of those parameters being utilized for simulations. Specifically, as seen, the maximum speed of the flywheel is $300 \mathrm{rpm}$. While for its speed control, each of its PID parameters is given a preliminary value first according to the open-loop gain and the system stability criteria, and then, those parameters are 


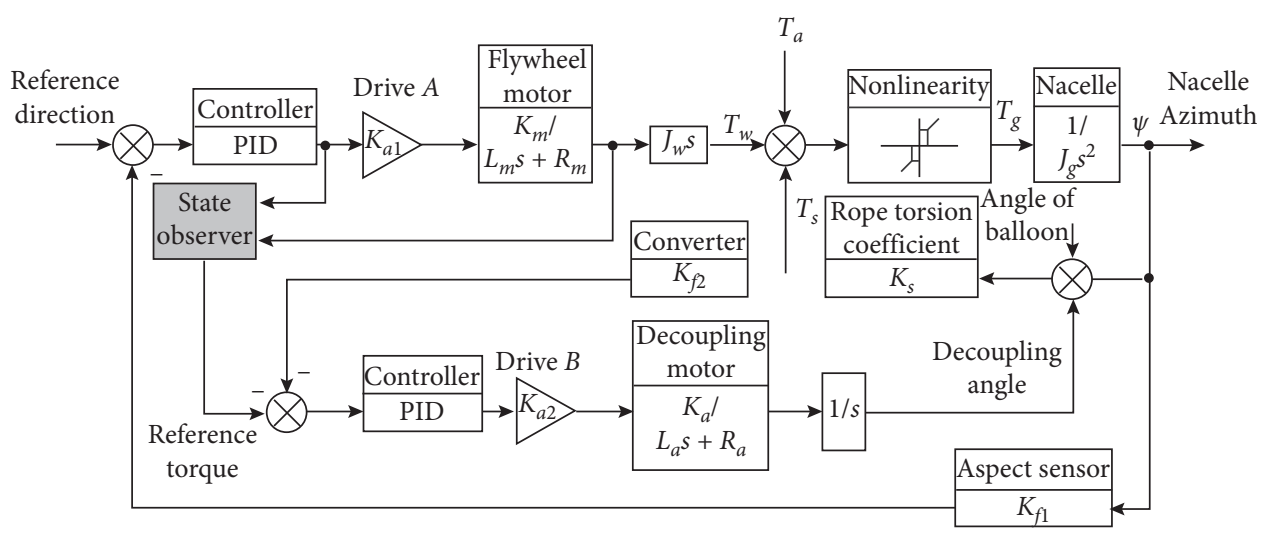

FIgURE 5: System schematic diagram of the proposed integrated decoupler.

TABLE 1: Simulation parameters.

\begin{tabular}{lc}
\hline Parameter & Value \\
\hline Gain of drive $A\left(K_{i 1}\right)$ & 0.8 \\
Rotor reactance of flywheel motor $\left(L_{m}\right)$ & $0.4 \Omega$ \\
Rotor impedance of flywheel motor $\left(R_{m}\right)$ & $1.7 \Omega$ \\
Equivalent inertia of flywheel $\left(J_{w}\right)$ & $3.0 \mathrm{~kg} \cdot \mathrm{m}^{2}$ \\
Equivalent inertia of gondola $(J)$ & $160 \mathrm{~kg} \cdot \mathrm{m}^{2}$ \\
Gain of drive $B\left(K_{i 2}\right)$ & 0.25 \\
Rotor reactance of decoupling motor $\left(L_{a}\right)$ & $1.1 \Omega$ \\
Rotor impedance of decoupling motor $\left(R_{a}\right)$ & $6.5 \Omega$ \\
The gain of azimuth sensor $\left(K_{f 1}\right)$ & 1.0 \\
Torque transformer coefficient & 0.1 \\
\hline
\end{tabular}

adjusted recursively according to the simulation results until they reach their optimal values. In our study, the PID parameters of flywheel speed control are $K_{p 1}=0.05$, $K_{i 1}=0.01$, and $K_{d 1}=0$, respectively. The same procedure is also applied for the decoupling motor, and the PID parameters for its output torque control are $K_{p 1}=0.5$, $K_{i 1}=0.02$, and $K_{d 1}=0$, respectively. The orientation reference angle of the gondola is set to be $150^{\circ}$.

As seen from equation (2) that once that $T_{s}$ and $T_{a}$ do not exist, the torque $T_{d}$ does not exist either. According to the simplified principle block diagram of azimuth control shown in Figure 3, the simulation curves of the azimuth direction and flywheel velocity of the balloon-borne gondola are shown in Figures 6(a) and 6(b), respectively, when ignoring $T_{s}$ and $T_{a}$. Simulation results demonstrated that the adjustment time of the azimuth direction of the balloonborne gondola is $2.5 \mathrm{~s}$, while the overshoot is $50 \%$, the flywheel velocity is finally stabilized to $0.05 \mathrm{rpm}$, and the stabilization time is $2.5 \mathrm{~s}$. Therefore, it can be known from the analysis that when there is no disturbance torque acting on the balloon-borne gondola, the orientation of the gondola and the rotation velocity of the flywheel will eventually stabilize without any decoupling mechanism, and the overshoot of the azimuth angle corresponds to the overshoot of the flywheel velocity. Such results also demonstrate that the simplified principle of azimuth control, as shown in
Figure 3, could meet the azimuth direction requirement of the balloon-borne gondola when there is no interference moment.

During the direction control process of the balloonborne gondola, the torsional moment $T_{s}$ and the uncertain torsional moment $T_{a}$ of the suspension rope will not disappear. Therefore, it can be known from equation (2) that $T_{d}$ exists. According to the simplified principle block diagram of azimuth control, as shown in Figure 3, either $T_{s}$ or $T_{a}$ is not ignored; hence, when simulation experiments are performed, the results for the azimuth direction and flywheel rotation velocity of the balloon-borne gondola are shown in Figures 7(a) and 7(b), respectively. Simulation results of the balloon-borne gondola cannot be stabilized to a given orientation, that is, the orientation of the balloon-borne gondola cannot be stabilized to $150^{\circ}$, while the velocity of the flywheel eventually becomes saturated. Therefore, it can be known from the analysis that when there is an interference torque acting on the balloon-borne gondola without a decoupling mechanism, the azimuth of the gondola is still in a controllable state before the flywheel rotation velocity is saturated. However, during the process when the flywheel velocity is increasing and becoming saturated, the azimuth angle entered an uncontrollable state. The above analysis shows that the simplified principle of azimuth control, as shown in Figure 3, cannot meet the azimuth orientation requirements of the balloon-borne gondola when there is an interference torque.

To address the above issue while to verify the effectiveness of our proposed scheme, the decoupler device is integrated into the control system of the balloon-borne gondola. According to the schematic diagram of the integrated decoupler, as shown in Figure 5, simulation experiments are performed when $T_{s}$ and $T_{a}$ are not ignored. The simulation results of the azimuth direction of the balloonborne gondola and the velocity of the flywheel are shown in Figures 8(a) and 8(b), respectively. When observing the simulation results, we can see that the adjustment time of the orientation of the balloon-borne gondola is $0.8 \mathrm{~s}$, the 


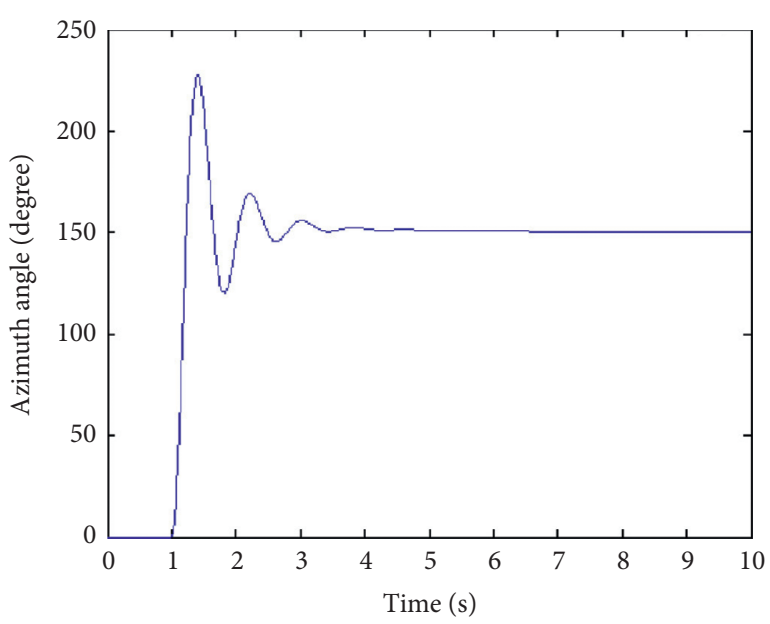

(a)

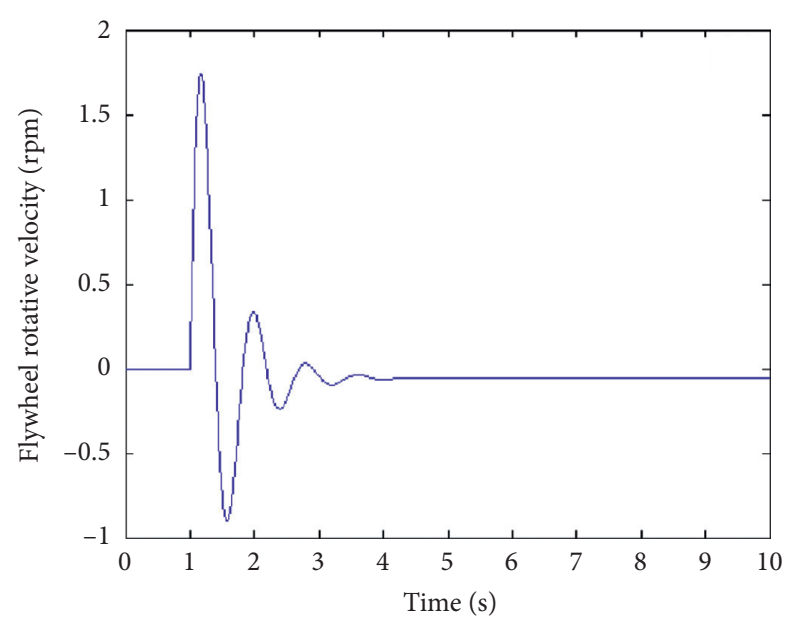

(b)

FIGURE 6: The simulated curves of the azimuth direction and flywheel speed of the balloon-borne gondola without disturbance torque and decoupling device: (a) the simulated azimuth angles at different times; (b) the simulated flywheel rotative velocity at different times.

overshoot is $19 \%$, the flywheel velocity is finally stabilized to $0.05 \mathrm{rpm}$, and the stabilization time is $1 \mathrm{~s}$. Therefore, it can be known from the analysis that when a decoupler is integrated, it can effectively restrain the influence of the coupling torque on the flywheel reactive azimuth control system and stabilize the flywheel velocity to around zero. In such a case, the influences of the coupling torque that is introduced by either the external interference torque or the cable torque could be reduced to a minimum, while there is no need for the flywheel motor to generate any additional torque to offset the influences caused by the coupling torque frequently. Meanwhile, the flywheel adjustment sensitivity could be largely enhanced, which thus helps improve the system performances for the azimuth control. Based on the above analysis and comparison between Figures 6 and 7, one can conclude that the control system incorporating the decoupler mechanism can solve the problem of the influence of the coupling torque on the ordinary reaction flywheel control system, and it could also help improve the system dynamic performances significantly. The control accuracy has also been significantly improved to meet the azimuth orientation requirements for the balloon-borne gondola in the presence of disturbance torque.

3.2. Experimental Results. Experiments have also been carried out to verify the proposed integrated decoupler design. Due to those trade secrets being involved, both the experimental configurations and the field images of the balloon gondola are not presented in the manuscript. In our experiments, both the related wind disturbances and interferences have also been considered according to the meteorological conditions, and they are all converted to be equivalent coupling moments. Due to the limited page length of the papers, however, those experimental conditions were not given in detail in our manuscript.

Figure 9 presents the flight altitude diagram of the balloon-borne gondola during its flight test, and the data frame is sent in a period of $200 \mathrm{~ms}$ during its flight. The time when the balloon was just launched is considered to be the starting point of time. During the rising process of the gondola, the azimuth control was started at 1 hour and 10 minutes, at which the height was about 19, 300 meters, and it took about 1 hour and 50 minutes for the gondola to rise to the experimental height, which was about 32,500 meters. The azimuth control was turned off after 2 hours and 20 minutes, that is, after the end of the experiment. It is worth noting that, due to the error code in the data transmission process, there appeared some burrs on the altitude diagram during the time $(15,000-20,000) \times 0.2$ s within the testing process.

Figure 10 is the azimuth error diagram of the balloonborne gondola during the test. As seen, before 1 hour and 10 minutes, that is, before $21,000 * 0.2 \mathrm{~s}$, since the azimuth control was not activated, the azimuth error of the balloonborne gondola appeared to be in a state of violent oscillation. In the time between 1 hour and 10 minutes and 2 hours and 20 minutes, that is, between $21,000 * 0.2 \mathrm{~s}$ and $42,000 * 0.2 \mathrm{~s}$, as the orientation control is activated, the orientation error of the balloon-borne gondola is approximately stable to be around ${ }^{0 \circ}$. After 2 hours and 20 minutes, the azimuth error of the balloon-borne gondola returned to the state of severe vibration again since the azimuth control system is turned off. It should be emphasized that, between $21,000 * 0.2 \mathrm{~s}$ and $33000 * 0.2 \mathrm{~s}$, because the balloon-borne gondola is in the rising stage, the external disturbance torque $T_{d}$ is relatively large, while the azimuth error curve between $33,000 * 0.2 \mathrm{~s}$ and $42,000 * 0.2 \mathrm{~s}$ is smoother. Such comparison analysis 


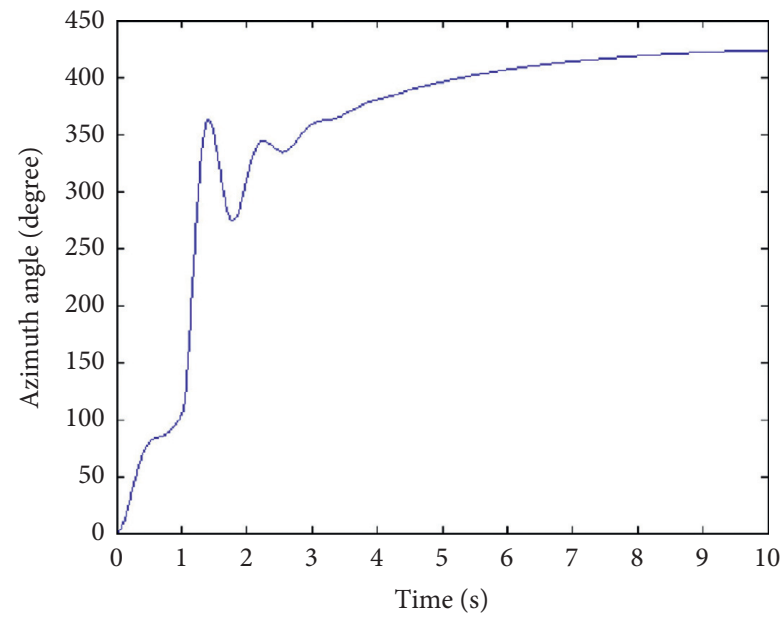

(a)

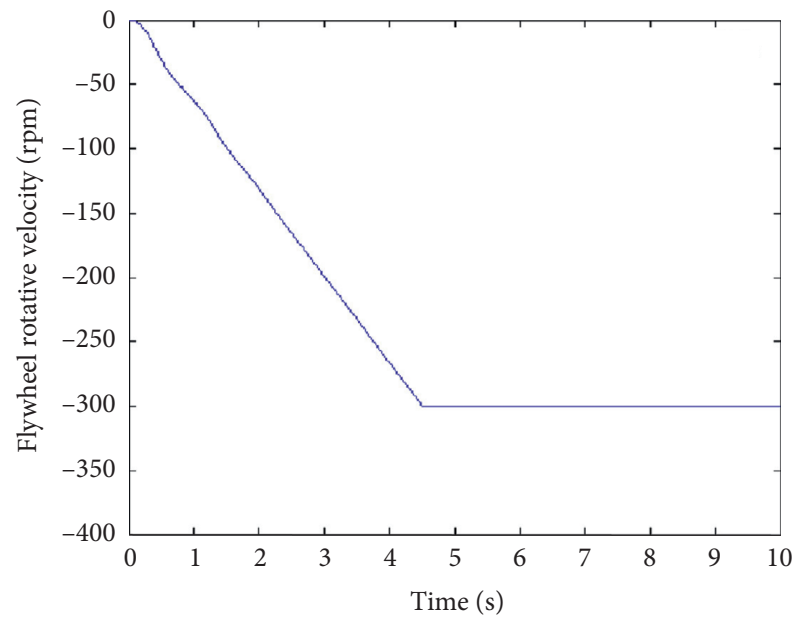

(b)

Figure 7: The simulated curves of the azimuth direction and flywheel speed of the balloon-borne gondola with an interference torque of $2.5 \mathrm{Nm}$ yet without the decoupling device: (a) the simulated azimuth angles at different times; (b) the simulated flywheel rotative velocity at different times.

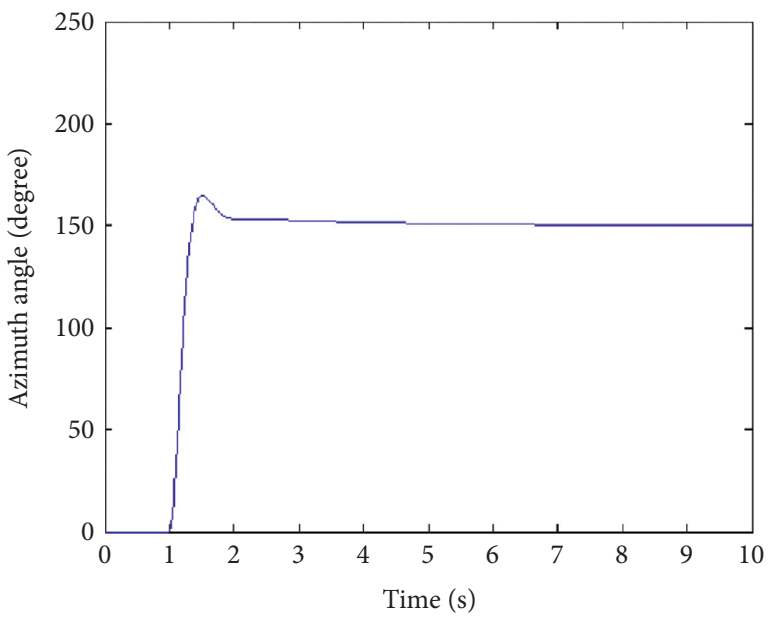

(a)

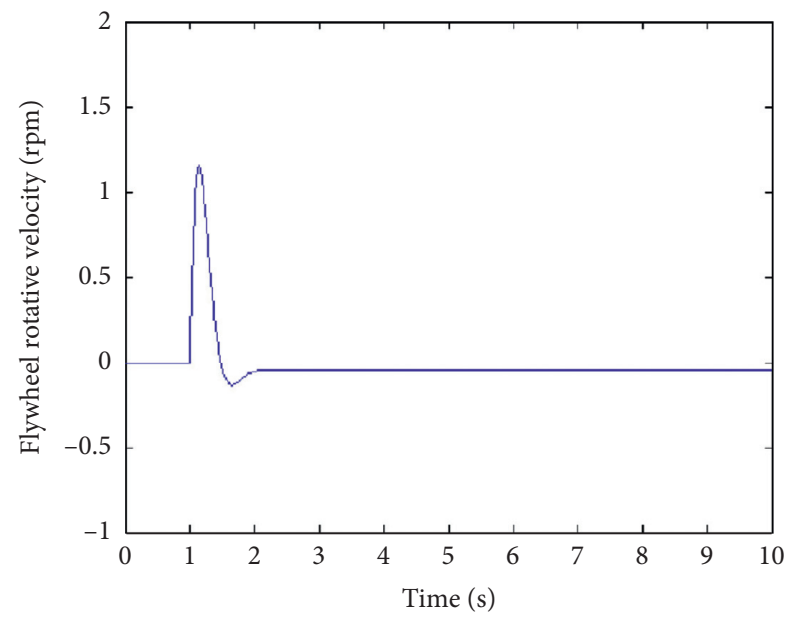

(b)

FIGURE 8: The simulated curves of the azimuth direction and flywheel speed of the balloon-borne gondola with an interference torque of $2.5 \mathrm{Nm}$ with the proposed decoupler: (a) the simulated azimuth angles at different times; (b) the simulated flywheel rotative velocity at different times.

demonstrates that the proposed integrated decoupler, as shown in Figure 5, could effectively suppress the influences of the coupling torque on the reactive position control of the flywheel in practice, and the performances of the direction control for the balloon-borne gondola can meet the requirements of azimuth. 


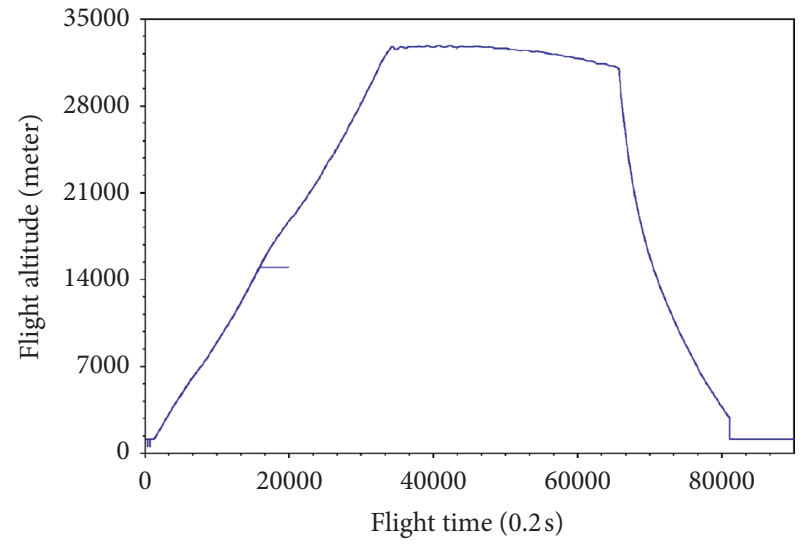

FIGURE 9: The measured flight height diagram of the balloon-borne gondola tested.

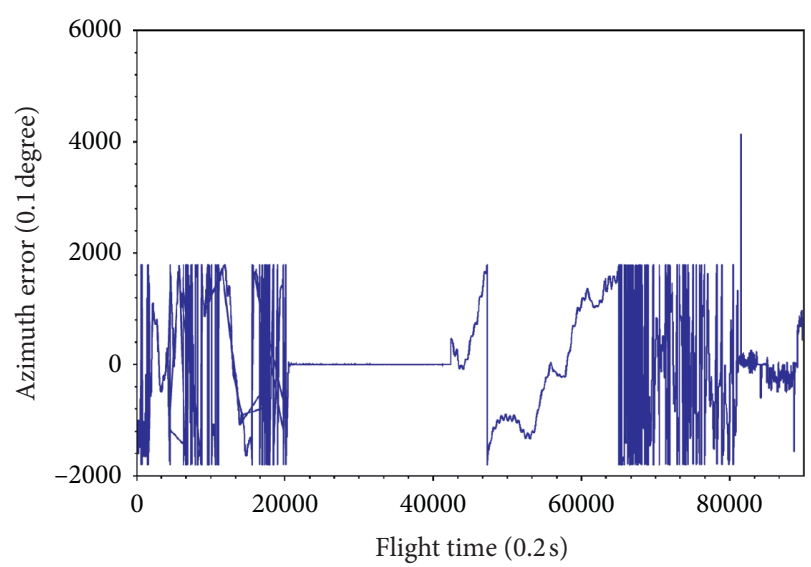

Figure 10: The deviation diagram of the balloon-borne gondola azimuth control.

\section{Conclusion}

To address the problem related to coupling torques caused by external disturbance, uncertainty factors, and system frictional nonlinearities within the azimuth control system of ball-borne gondola, a new type of integrated decoupler device is proposed and implemented in this study. Both simulations and experiments have been conducted to verify the effectiveness of the proposed decoupler. The results show that such a device could effectively suppress the influence of the coupling torque on the angular momentum exchange device enabling it to work at the optimal working point. Meanwhile, it could also quickly sense and eliminate the coupling torque caused by uncertain factors to prevent them from affecting the azimuth control system. Finally, such a decoupler can also compensate the coupling torque caused by the frictional nonlinearity of the control system structure so that the available control torque of the azimuth control system can be extracted more timely, thereby improving the control accuracy and agility of the system. Such a decoupler design can also be applied to other control systems using angular momentum exchange devices as actuators and, thus, may have broad potential applications. Our future work would focus on two main aspects. On the one hand, it is expected that the system output power and integration could be further improved, while its size could be minimized such that it could be applied onto some other systems, and on the other hand, more robust and flexible control strategies that could be integrated into the system are highly desired to further improve its control performances.

\section{Data Availability}

Both the simulation and experimental data used to support the findings of this study are available from the corresponding author upon request.

\section{Disclosure}

All funding agencies had no role in the collection, analysis, or interpretation of the data or in the decision to submit the manuscript for publication.

\section{Conflicts of Interest}

The authors declare that they have no conflicts of interest.

\section{Acknowledgments}

This research was supported in part by the Fundamental Research Funds for the Central Universities (Grant no. G2018KY0308), China Postdoctoral Science Foundation (Grant no. 2018M641013), and Postdoctoral Science Foundation of Shaanxi Province (Grant no. 2018BSHYDZZ05).

\section{References}

[1] M. Barbary and P. Zong, "Optimisation for stealth target detection based on stratospheric balloon-borne netted radar system," IET Radar, Sonar \& Navigation, vol. 9, no. 7, pp. 802-816, 2015.

[2] L. J. Romualdez, C. J. Damaren, L. Li et al., "Precise pointing and stabilization performance for the balloon-borne imaging testbed: 2015 test flight," Proceedings of the Institution of Mechanical Engineers, Part G: Journal of Aerospace Engineering, vol. 231, no. 4, pp. 713-727, 2017.

[3] P. C. Nagler, B. Edwards, B. Kilpatrick et al., "Observing exoplanets in the near-infrared from a high altitude balloon platform," 2019.

[4] A. Guetta, R. Amber, and Y. Guetta, "Balloon-borne platform stabilization," 2017.

[5] D. Huguenin, "Design and performance of stratospheric balloon-borne platforms for infrared astrophysical observations," Infrared Physics \& Technology, vol. 35, no. 2-3, pp. 195-202, 1994.

[6] T. Villela, R. A. Fonseca, P. De Souza et al., "Development of an attitude control system for a balloon-borne gamma ray telescope," Advances in Space Research, vol. 26, no. 9, pp. 1415-1418, 2000.

[7] X. Ye, N. Yajima, G. Ai, and N. Hu, "Attitude control and high precision pointing control system of a large balloon borne solar telescope," Advances in Space Research, vol. 26, no. 9, pp. 1419-1422, 2000. 
[8] A. Aboobaker, P. Ade, D. Araujo et al., "The ebex balloonborne experiment gondola, attitude control, and control software," The Astrophysical Journal Supplement Series, vol. 239, no. 1, p. 9, 2018.

[9] A. Doi, Y. Kono, K. Kimura et al., "A balloon-borne very long baseline interferometry experiment in the stratosphere: systems design and developments," Advances in Space Research, vol. 63, no. 1, pp. 779-793, 2019.

[10] M. Martorella and E. Aboutanios, "Balsar: a stratospheric balloon-borne sar system," in Advanced Technologies for Security Applications, pp. 283-294, Springer, Berlin, Germany, 2020.

[11] J. Romualdez, Design, implementation, and operational methodologies for sub-arcsecond attitude determination, control, and stabilization of the super-pressure balloon-borne imaging telescope (superbit), Ph.D. dissertation, Springer, Berlin, Germany, 2017.

[12] L. Cao, B. Xiao, and M. Golestani, "Robust fixed-time attitude stabilization control of flexible spacecraft with actuator uncertainty," Nonlinear Dynamics, vol. 63, pp. 1-15, 2020.

[13] E. A. Chaffee, I. S. Smith, J. Noll, and B. Martin, "The investigation and measurement of balloon dynamics at the apex and base of a scientific balloon," in Proceedings of the AIAA Balloon Systems Conference, p. 3286, New York, NY, USA, 2017.

[14] F. Aubin, B. Bayman, S. Hanany et al., "Torsional balloon flight line oscillations: comparison of modelling to flight data," Advances in Space Research, vol. 60, no. 3, pp. 702-708, 2017.

[15] Q. Sun, K.-M. Lim, H. P. Lee, and B. C. Khoo, "Air drag on a stratospheric balloon in tropical regions," 2015.

[16] M. Ruderman and M. Iwasaki, "Observer of nonlinear friction dynamics for motion control," IEEE Transactions on Industrial Electronics, vol. 62, no. 9, pp. 5941-5949, 2015.

[17] T. N. Do, T. Tjahjowidodo, M. W. S. Lau, and S. J. Phee, "Nonlinear friction modelling and compensation control of hysteresis phenomena for a pair of tendon-sheath actuated surgical robots," Mechanical Systems and Signal Processing, vol. 60-61, pp. 770-784, 2015.

[18] M. Eom and D. Chwa, "Robust swing-up and balancing control using a nonlinear disturbance observer for the pendubot system with dynamic friction," IEEE Transactions on Robotics, vol. 31, no. 2, pp. 331-343, 2015.

[19] J. V. H. de Lorenzo, S. A. Rinehart, M. Rizzo et al., "Flight performance of the attitude control system of the balloon experimental twin telescope for infrared interferometry (bettii)," in Ground-based and Airborne Telescopes VIIVol. 10700, International Society for Optics and Photonics, Berlin, Germany, 2018.

[20] P. Olejnik and J. Awrejcewicz, "Coupled oscillators in identification of nonlinear damping of a real parametric pendulum," Mechanical Systems and Signal Processing, vol. 98, pp. 91-107, 2018.

[21] L. Zeng, Y. Zang, Z. Gao, K. Liu, and X. Liu, "Stability analysis of the rolling mill multiple-modal-coupling vibration under nonlinear friction," Journal of Vibroengineering, vol. 17, no. 6, 2015.

[22] T. Nakano, "Design of 'attitude control system for stratospheric balloon gondolas by sliding mode control," in IOP Conference Series: Materials Science and Engineeringvol. 501, no. 1, IOP Publishing, Bristol, UK, 2019.

[23] B. Xiao, L. Cao, S. Xu, and L. Liu, "Robust tracking control of robot manipulators with actuator faults and joint velocity measurement uncertainty," IEEE/ASME Transactions on Mechatronics, Berlin, Germany, 2020.

[24] M. Safonova, K. Nirmal, A. Sreejith et al., "Measurements of gondola motion on a stratospheric balloon flight," 2016.

[25] M. Saccoccio, J.-P. Bernard, Y. André et al., "Operations and results of the pilot balloon borne telescope flight," in Proceedings of the 14th International Conference on Space Operations, p. 2612, New York, NY, USA, 2016.

[26] A. S. Rahlin, The first flight of the spider balloon-borne telescope, Ph.D. dissertation, Princeton University, New Jersey, NJ, USA, 2016.

[27] N. Peyret, M. Rosatello, G. Chevallier, and J.-L. Dion, "A mindlin derived dahl friction model," Mechanism and Machine Theory, vol. 117, pp. 48-55, 2017.

[28] P. F. L. Marques, P. Flores, and H. M. Lankarani, "Sensitivity analysis of friction force model parameters in multibody dynamics," in Proceedings of the 4th Joint International Conference on Multibody System Dynamics, New York, NY, USA, 2016.

[29] D. Zhang, J. Zhou, and W. Huang, "Design of decoupling control system for the azimuth control of balloon-borne mission," in Proceedings of the 2016 35th Chinese Control Conference (CCC), IEEE, Berlin, Germany, pp. 10814910 819, 2016.

[30] S. Gurubaran, M. Shanmugam, K. Jawahar, K. Emperumal, P. Mahavarkar, and S. K. Buduru, "A high-altitude balloon experiment to probe stratospheric electric fields from low latitudes," 2017.

[31] M. Chauvin, H.-G. Florén, M. Jackson et al., "The design and flight performance of the pogolite pathfinder balloon-borne hard X-ray polarimeter," Experimental Astronomy, vol. 41, no. 1-2, pp. 17-41, 2016. 